\title{
Potentiel de photosynthèse hivernale du douglas (Pseudotsuga menziesii Mirb.) en relation avec le régime thermique
}

\author{
J.-M. GUEHL \\ avec la collaboration technique de P. Gross \\ I.N.R.A., Station de Sylviculture et de Production \\ Centre de Recherches forestières de Nancy \\ Champenoux, $F 54280$ Seichamps
}

\begin{abstract}
Résumé
Cet article est une contribution à l'étude des potentialités photosynthétiques hivernales des conifères en zone tempérée.

Il met en évidence une action du régime thermique sur l'activité photosynthétique du douglas à deux niveaux d'échelle dans le temps. A l'échelle instantanée, la réponse photosynthétique à la température se caractérise par un large domaine optimal plutôt que par un optimum marqué. A l'échelle de la décade, la capacité photosynthétique oscille en permanence sous l'action d'un jeu d'endommagement - réparation de la fonction photosynthétique liée à l'alternance de périodes de gel et de dégel.

A l'approche du débourrement, notre étude permet de relier les modifications intervenant dans les échanges gazeux de $\mathrm{CO}_{2}$ au niveau du rameau au développement des bourgeons.
\end{abstract}

\section{1. - Introduction}

Le caractère sempervirent de la quasi-totalité des conifères de la zone tempérée confère-t-il à ces derniers un fonctionnement photosynthétique à contribution hivernale significative?

Dans un contexte climatique à froid hivernal prononcé caractérisant les régions tempérées continentales septentrionales (ZELAWSKI \& GoRAL, 1966; ZELAWSKI \& KuCHarska, 1967) ou les zones de montagne (PISEK \& WinkLer, 1958 ; BamberG et al., 1966; Schwarz, 1971; Hagihara \& Hozumi, 1977; Tranquillini, 1979), l'activité photosynthétique hivernale des conifères est fortement réduite ou inexistante.

A l'opposé, sous le climat à hiver très doux des îles britanniques, Fry \& PHILLIPS (1977) ont mis en évidence une évolution saisonnière de l'activité photosynthétique de plusieurs espèces (Abies grandis, Picea sitchensis et Tsuga heterophylla) ne présentant qu'une dépression hivernale moindre. 
Entre ces deux extrêmes, sous un climat tel celui régnant sur une grande partie de la France, caractérisé par un hiver dont les phases de gel sont entrecoupées de périodes de températures positives, les résultats obtenus (SchmidT-VogT \& Gross, 1976 ; KüNSTle \& Mitscherlich, 1978) restent trop ponctuels pour que puisse être effectuée une analyse de la productivité photosynthétique hivernale des conifères.

Le présent article tente d'apporter quelques éléments de base pour une telle analyse à partir de la description du fonctionnement photosynthétique hivernal du Douglas (Pseudotsuga menziesii Mirb. Franco.) en relation avec le régime thermique naturel. Le Douglas, dont un grand nombre de provenances sont originaires de la frange côtière pacifique de l'Amérique du Nord, caractérisée par des hivers doux permettant une assimilation carbonée hivernale importante (EMMINGHAM \& WARING, 1977), a été très largement introduit en France. Cette extension artificielle de l'aire de répartition risque-t-elle de s'accompagner parfois d'une diminution des potentialités de photosynthèse hivernale? En France, des différences de photosynthèse hivernale en relation avec des variations climatiques régionales peuvent-elles être à l'origine de différences dans les productions ligneuses?

\section{2. - Matériel et méthodes}

\subsection{Le matériel végétal utilisé}

Les mesures de photosynthèse ont été effectuées sur des rameaux prélevés sur des arbres d'une plantation de Douglas (Pseudotsuga menziesii Mirb., provenance SEAquest dans l'Etat du Washington aux U.S.A., latitude $48^{\circ} 25$, longitude $121^{\circ} 35$, altitude $100 \mathrm{~m}$ ), âgée de 19 ans et située à proximité du Centre National de Recherches Forestières de Champenoux, à $15 \mathrm{~km}$ à l'Est de Nancy. Quatre arbres de hauteur moyenne $10,5 \mathrm{~m}$ ont été choisis sur la lisière sud du peuplement dont AussENAC (1980) donne une description précise.

\subsection{Méthode expérimentale}

Au début de la matinée de chaque journée de mesure, deux rameaux de pleine lumière, exposés au sud et situés entre 2 et $3 \mathrm{~m}$ du sol, ont été coupés sur chacun des quatre arbres. La section des huit rameaux prélevés a été immédiatement placée sous eau pour ne pas interrompre leur alimentation hydrique. Amenés au laboratoire, les rameaux ont été recoupés sous eau afin de ne conserver que la partie centrale de la dernière pousse (1978). Sans sortir de l'eau, la section des rameaux a été introduite dans des tubes eux-mêmes remplis d'eau (2 tubes recevant chacun quatre rameaux).

\subsection{Mesure des échanges gazeux de $\mathrm{CO}_{2}$}

Après imperméabilisation des tubes, les rameaux ont été placés dans une chambre d'assimilation s'intégrant dans un système ouvert de mesure des échanges gazeux de $\mathrm{CO}_{2}$. L'air prélevé à l'extérieur traversait le système à raison de $60 \mathrm{lh}^{-1}$. La concentration en $\mathrm{CO}_{2}$ de l'air à l'entrée et à la sortie de la chambre a été mesurée à l'aide d'un analyseur de gaz par absorption infra-rouge (HARTMANN \& Braun, type Uras 2) utilisé en mesure absolue. 
Le dispositif de mesure n'étant pas muni d'un système de régulation de la concentration en $\mathrm{CO}_{y}$, les valeurs $\mathrm{F}_{\mathrm{X} \text { i }}$ de la photosynthèse mesurées à une concentration c (*) ont été ramenées à une concentration standard de $300 \mathrm{vpm}$ (volumes par million) de $\mathrm{CO}_{2}$ suivant la formule (1) exprimant la relation linéaire croissante entre la photosynthèse nette et la concentration en $\mathrm{CO}_{2}$ de l'air existant pour les plantes de type $C_{3}$ en conditions d'éclairement non limitantes et des concentrations de $\mathrm{CO}_{2}$ dans l'air inférieures ou égales aux concentrations atmosphériques normales (CHARTIER \& BETHENOD, 1977).

$$
F_{X 3111}=\frac{300-I^{\top}}{C-I^{\top}} \cdot F_{N c}
$$

I : valeur du point de compensation de la photosynthèse pour le $\mathrm{CO}_{2}$ dans les conditions de mesure. Conformément aux résultats d'une expérimentation préalable (GueHı, 1978), nous avons adopté une valeur de $60 \mathrm{vpm}$ pour $\Gamma$.

$\mathrm{F}_{\mathrm{X} \text {. }} \quad$ : intensité de photosynthèse nette mesurée à une concentration de $\mathrm{CO}_{2}$ dans lair égale à $\mathrm{C}$ vpm.

$\mathrm{F}_{\mathrm{X} \text { зиm }}$ : intensité théorique de la photosynthèse nette pour une concentration de $\mathrm{CO}_{2}$ dans l'air égale à $300 \mathrm{vpm}$.

La photosynthèse nette des rameaux a été rapportée à l'unité de surface foliaire simple mesurée à l'aide d'un planimètre Milliplan (Société Métraplan S.P.A.A.) par lintermédiaire d'une photographie contact des aiguilles (papier photographique Kodabrom $\mathrm{G} 5$ à fort contraste). Le poids de matière sèche des aiguilles $\left(\mathrm{MS}_{\mathrm{A}}\right)$, des bourgeons $\left(\mathrm{MS}_{13}\right)$ ainsi que des rachis des rameaux $\left(\mathrm{MS}_{\mathrm{R}}\right)$ ont également été déterminés (tableau 1).

\subsection{Contrôle des facteurs physiques de la photosynthèse}

\subsection{Température}

La chambre d'assimilation a été placée dans une enceinte réfrigérée d'intensité frigorifique réglable et dont la paroi faciale est constituée par une plaque de plexiglas afin de permettre l'éclairement des rameaux. La température de l'air dans l'enceinte d'assimilation a été mesurée à l'aide d'un thermocouple Cuivre/Constantan abrité du rayonnement de la lampe. Après la mise en place de la chambre et à l'obscurité, la température de l'air a été progressivement abaissée à $4{ }^{\circ} \mathrm{C}$ en trois heures.

\subsection{Eclairement}

Une lampe à vapeur de sodium haute pression (Philips Sont, 400 watts) a alors été allumée, et fournissait au niveau des rameaux un flux de photons de densité 280 «Einsteins $\mathrm{m}^{-2} \mathrm{~s}^{-1}$ mesuré à l'aide d'un «Lambda quantum sensor ", dans la bande spectrale des radiations photosynthétiquement actives $(400-700 \mathrm{~nm})$. Ces conditions d'éclairement peuvent être considérées comme saturantes pour la photosynthèse du Douglas (Gueht, 1978), du moins en hiver.

(*) Dans certains cas, l'activité assimilatrice des rameaux a pu abaisser la concentration en $\mathrm{CO}_{\mathfrak{z}}$ à l'intérieur de la chambre d'assimilation de $100 \mathrm{vpm}$ par rapport à l'extérieur. 


\section{Tableau 1}

Surface foliaire simple (SF) et poids de matière sèche (MS)

des différentes parties des rameaux de douglas utilisés pour les mesures de photosynthèse du 16-2-79 au 16-5-79

Single needle area (SF) and dry-weight (MS) of the different parts of the Douglas-fir shoots used for photosynthesis measurements from 2-16th-79 to 5-16th-79

\begin{tabular}{|c|c|c|c|c|c|c|c|}
\hline \multicolumn{2}{|c|}{ Date } & $\begin{array}{c}\mathrm{SF} \\
\left(\mathbf{d m}^{2}\right)\end{array}$ & $\begin{array}{c}\mathrm{MS}_{\Lambda} \\
(\mathrm{g})\end{array}$ & $\begin{array}{c}\mathrm{MS}_{1 k} \\
(\mathrm{~g})\end{array}$ & $\begin{array}{c}\mathrm{MS}_{\mathrm{LR}} \\
(\mathrm{g})\end{array}$ & $\frac{\mathrm{MS}_{\mathrm{A}}}{\mathrm{SF}}$ & $\frac{\mathrm{MS}_{\mathrm{B}}}{\mathrm{MS}_{\mathrm{B}}}$ \\
\hline $16-02-79$ & $\ldots$ & 2,94 & 4,80 & 0,72 & 2,19 & 1,63 & 0,33 \\
\hline $21-02-79$ & $\ldots \ldots$ & 3,12 & 4,66 & 0,55 & 2,03 & 1,49 & 0,27 \\
\hline $26-02-79$ & $\ldots \ldots$ & 3,38 & 5,17 & 0,70 & 2,34 & 1,53 & 0,30 \\
\hline $28-02-79$ & $\ldots$ & 2,97 & 5,16 & 0,98 & 3,19 & 1,74 & 0,30 \\
\hline $12-03-79$ & $\ldots$ & 3,52 & 5,49 & 0,75 & 2,70 & 1,56 & 0,28 \\
\hline $16-03-79$ & $\ldots \ldots$ & 3,12 & 4,51 & 0.50 & 1,81 & 1,45 & 0,28 \\
\hline $20-03-79$ & $\ldots$ & 3,19 & 4,80 & 0,40 & 1,81 & 1,50 & 0,22 \\
\hline $27-03-79$ & $\ldots \ldots$ & 3,14 & 5,02 & 0,97 & 2,53 & 1,60 & 0,38 \\
\hline 02-04-79 & $\ldots \ldots \ldots$ & 3,34 & 4,67 & 0,79 & 2,07 & 1,40 & 0,38 \\
\hline $07-04-79$ & $\ldots \ldots$ & 2,93 & 4,04 & 0,64 & 1,68 & 1,38 & 0,38 \\
\hline $12-04-79$ & $\ldots \ldots \ldots$ & 2.43 & 3,72 & 1,09 & 2,20 & 1,53 & 0,49 \\
\hline $19-04-79$ & $\ldots \ldots \ldots$ & 2,61 & 4,55 & 1,23 & 2,46 & 1,74 & 0,50 \\
\hline $25-04-79$ & $\ldots \ldots$ & 2,01 & 3,73 & 1,12 & 2,14 & 1,85 & 0,52 \\
\hline 04-05-79 & . & 2,20 & 3,70 & 1,44 & 2,11 & 1,68 & 0,68 \\
\hline 09-05-79 & $\ldots$ & 1,72 & 3,26 & 1,03 & 2,07 & 1,90 & 0,50 \\
\hline $15-05-79$ & $\ldots \ldots$ & 2,10 & 3,61 & 1,71 & 2,14 & 1,72 & 0,80 \\
\hline $16-05-79$ & $\ldots \ldots$ & 1,97 & 3,66 & 1,61 & 1,79 & 1,86 & 0,90 \\
\hline
\end{tabular}

$\mathrm{MS}_{\mathrm{A}}$ : Poids de matière sèche des aiguilles.

Needle dry-weight.

$\mathrm{MS}_{\mathrm{I}}$ : Poids de matière sèche des bourgeons. Bud dry-weight.

MS $_{\text {Ir }}$ : Poids de matière sèche des rachis. Shoot-axis dry-weight.

En réduisant graduellement l'intensité frigorifique de l'enceinte réfrigérée, des températures de l'air de $8{ }^{\circ} \mathrm{C}$ à $24{ }^{\circ} \mathrm{C}$ ont pu être obtenues pour les mesures de photosynthèse. La durée d'établissement des états d'équilibre thermique et de concentration en $\mathrm{CO}_{2}$ à la sortie de la chambre d'assimilation était environ dune heure par point de mesure.

\subsection{Facteurs hydriques}

L'humidité de l'air à l'intérieur de la chambre d'assimilation n'a été l'objet d'aucun contrôle. Par suite de la transpiration des rameaux, cette humidité a donc constamment été supérieure à l'humidité atmosphérique. Par ailleurs, les observations faites par un certain nombre d'auteurs sur les conditions d'utilisation des rameaux de conifères 
(Larcher, 1963 ; Neilson et al., 1972; Guehl, 1978; Aussenac \& Ducrey, communication personnelle) coupés tendent à prouver qu’à conditions expérimentales fixées :

- la photosynthèse des rameaux coupés initialement bien alimentés en eau n'est pas affectée par la section à condition de bien maintenir les extrémités coupées dans l'eau;

- après la section, aucune perturbation ne se manifeste dans un intervalle de temps au moins égal aux huit heures de la durée de nos mesures.

La méthode des rameaux coupés donne donc accès à un potentiel de photosynthèse caractérisant l'optimisation des facteurs hydriques au moment de la mesure.

\section{3. - Résultats}

\subsection{Evolution de l'activité photosynthétique des rameaux de Douglas}

Les mesures de photosynthèse ont été effectuées au cours d'une période hivernale s'étendant du 16 février 1979 au 16 mai 1979. Les figures $1 \mathrm{a}, \mathrm{b}, \mathrm{c}$ et d présentent l'ensemble des 17 courbes de réponse de la photosynthèse nette des rameaux de Douglas à la température régnant à l'intérieur de la chambre d'assimilation. L'allure générale de la réponse photosynthétique à la température est marquée par l'existence d'un optimum peu marqué à l'intérieur du domaine thermique exploré.

Mais le trait dominant qui se dégage de l'examen des courbes de la figure 1 est celui d'une grande variabilité de la photosynthèse dans le temps. Schématiquement cette variabilité se traduit par une translation des courbes le long de l'axe des ordonnées. Une confrontation rapide de ce jeu de translation (figure 1) aux températures journalières mesurées sous abri à proximité de la plantation étudiée (figure 2) montre que les périodes de chute importante de l'activité photosynthétique (du 16 février au 28 février, du 27 mars au 7 avril, du 25 avril au 4 mai) correspondent à des périodes de températures minimales négatives. A l'inverse, les phases d'augmentation de l'activité photosynthétique (du 28 février au 16 mars, du 7 avril au 12 avril, du 19 avril au 25 avril) caractérisent les périodes de températures minimales positives. Cette première observation suggère une analyse des résultats sous l'angle des effets rémanents du régime thermique naturel sur l'activité photosynthétique.

\subsection{Effets rémanents du régime thermique sur le fonctionnement photosynthétique du Douglas}

\subsection{Capacité photosynthétique des rameaux de Douglas}

Les conditions expérimentales dans lesquelles ont été effectuées les mesures de photosynthèse nous ont amenés à formuler les hypothèses suivantes :

a) Les facteurs hydriques ne sont pas limitants pour la photosynthèse au moment de la mesure (2.43). 

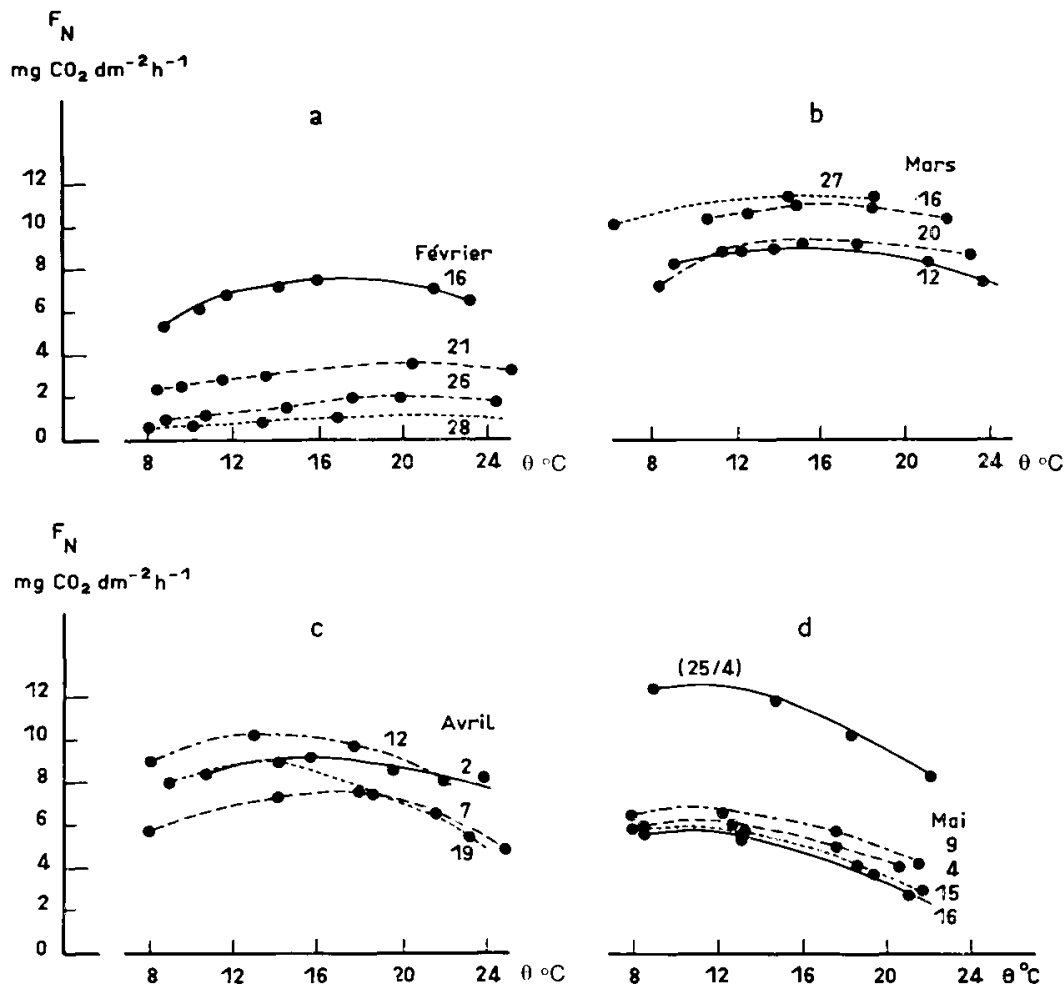

FiG. 1

Réponse de la photosynthèse nette $\left(F_{*}\right)$ à la température de l'air (0)

à différentes dates du 16-2-79 au 16-5-79. Cette dernière date correspond au débourrement

Response of net photosynthesis $\left(F_{N}\right)$ to air temperature $(\theta)$ at different days from 2-16th-79 to 5-16th-79. Bud sprouting occured on that last date

b) L’éclairement est saturant pour la photosynthèse (2.42).

A cette double optimisation de facteurs «actuels » de la photosynthèse s'ajoute:

c) Les valeurs de photosynthèse correspondent toutes à une même concentration en $\mathrm{CO}_{22}$ de l'air égale à 300 vpm (2.3).

Dans ces conditions, pour chacune des courbes de la figure 1, la valeur maximale $F_{X \text { MAX }}$ de la photosynthèse nette correspond à la limite supérieure de l'intensité photosynthétique imposée par l'état physiologique des rameaux à une concentration en $\mathrm{CO}$. de l'air égale à $300 \mathrm{vpm}$. Indépendante des facteurs environnementaux «actuels», cette capacité photosynthétique $F_{X M A X}$ présente un intérêt évident pour l'étude des effets rémanents du climat sur la photosynthèse. 


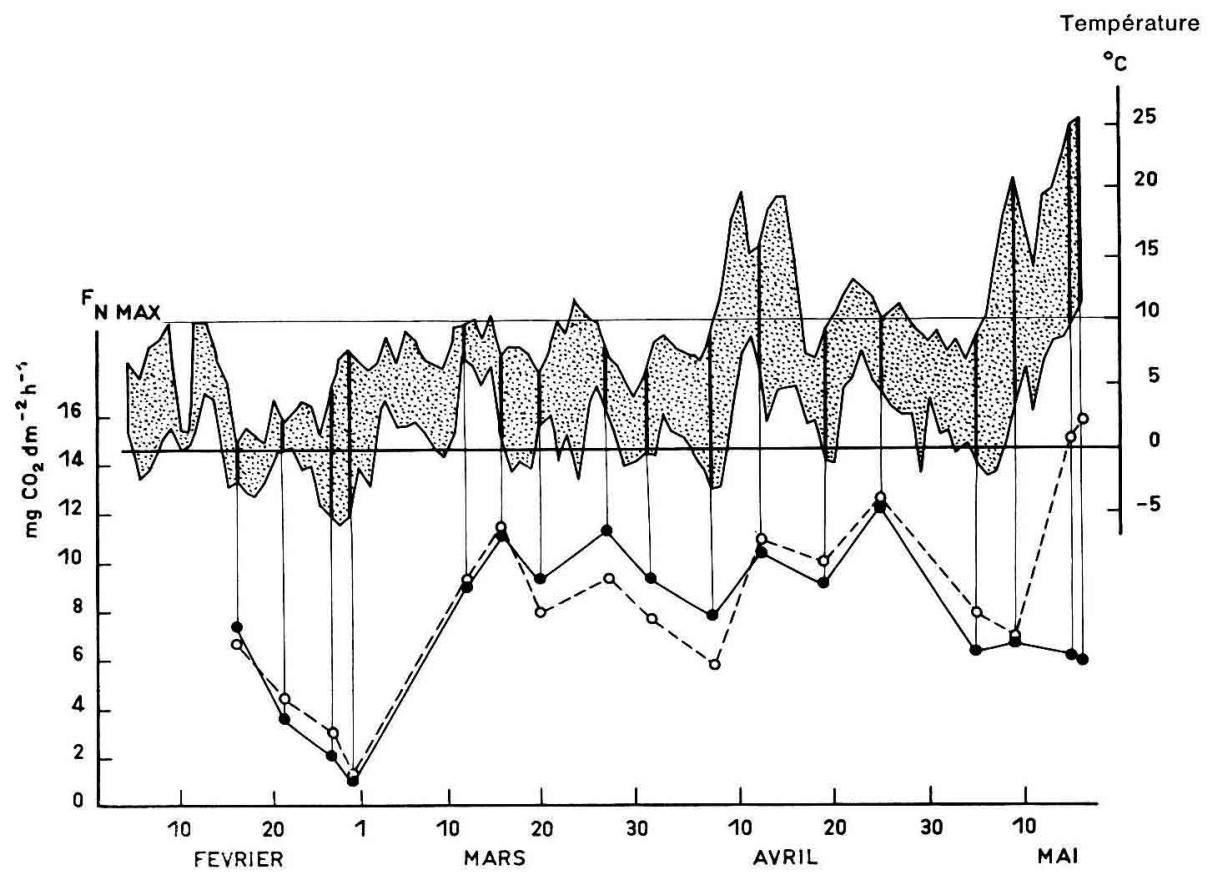

FiG. 2

Evolution de la capacité photosynthétique du 16-2-79 au 16-5-79 en relation avec le régime thermique naturel. Le 16-5-79 correspond au débourrement

Changes of the photosynthetic capacity from 2-16th-79 to 5-16th-79

related to the outside temperature regime. Bud sprouting occured on 5-16th-79

En haut : températures journalières minimales et maximales relevées sous abri.

En bas : évolution de la capacité photosynthétique.

$(\bullet-\bullet)$ : valeurs mesurées $\left(F_{x} \mu_{x}\right) ;\left(O-O_{0}\right)$ : valeurs calculées ( $\hat{F}_{x}$ Mx $)$ à l'aide d'un modèle mathématique d'après l'histoire thermique des rameaux [cf. 3.222 ; équation (3)].

Upper part : Daily minima and maxima temperatures.

Lower part: Changes of the photosynthetic capacity.

$(\bullet-\bullet)$ : measured values $\left(F_{X} y_{A X}\right) ;\left(O_{-} \bigcirc\right)$ : values predicted $\left(\vec{F}_{N} y_{A X}\right)$ on the basis of the thermal history of the shoots [see 3.222, equation (3)].

\subsection{Capacité photosynthétique et histoire thermique des rameaux}

\subsection{Description générale}

La courbe en trait plein de la partie inférieure de la figure 2 représente l'évolution de la capacité photosynthétique $\mathrm{F}_{\mathrm{N} \text { м } \Lambda \mathrm{X}}$ au cours de la période étudiée. Les températures journalières minimales et maximales de cette période apparaissent dans la partie supérieure de la figure 2. 
Quatre phases peuvent être distinguées dans l'évolution de la capacité photosynthétique :

- du 16 février au 28 février, on note une diminution importante de la capacité photosynthétique simultanément à l'occurrence du creux thermique le plus accentué de la période de mesure;

- le retour à une période pratiquement sans gel ( $-0,4{ }^{\circ} \mathrm{C}$ toutefois le 10 mars) du 3 mars au 16 mars s'accompagne d'une rapide remontée de lactivité photosynthétique ;

- du 16 mars au 9 mai, on note une variation en dent de scie de la capacité photosynthétique avec cependant une relative stabilisation autour d'une valeur égale à $10 \mathrm{mg} \mathrm{CO}_{2} \mathrm{dm}^{-2} \mathrm{~h}^{-1}$. Les valeurs les plus faibles de $\mathrm{F}_{\mathrm{N} \text { MAX }}$ correspondent aux creux thermiques ou en sont précédées de peu, alors que les valeurs les plus élevées correspondent aux crêtes thermiques;

- du 9 mai au 16 mai, la capacité photosynthétique accuse une légère diminution malgré une augmentation importante des températures. Au 16 mai, on se situe au stade $b_{3} d u$ développement des bourgeons (aiguilles de la nouvelle pousse partiellement libérées).

\subsection{Analyse statistique}

Afin de préciser certaines caractéristiques de la liaison existant entre $F_{N} \max$ et l'histoire thermique des rameaux, une analyse statistique des résultats a été effectuée. Le tableau 2 indique les coefficients de corrélation $\left(\mathrm{r}^{2}\right)$ des régressions linéaires

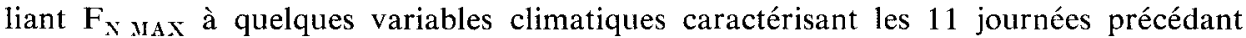

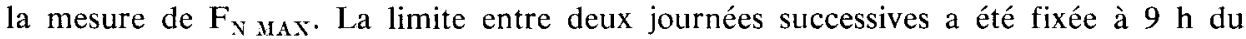
matin, heure de prélèvement des rameaux. Les mesures des 15 et 16 mai 1979 n'ont pas été prises en compte dans cette analyse. Pour ces deux dates, la proximité du débourrement semble s'accompagner d'une perturbation du déterminisme essentiellement climatique de la photosynthèse caractérisant la période antérieure.

$F_{X \text { max }}$ est significativement (seuil de 5 p. 100) relié à la température minimale de chacune des quatre journées précédant la mesure (colonne $T_{\text {MIN i }}$ du tableau 2). La meilleure relation $\mathrm{r}^{2}=0,62$ est obtenue pour la troisième journée avant la mesure $(i=3)$. Cette dernière observation est également valable dans le cas des températures journalières maximales et moyennes $\left(2^{\circ}\right.$ et $3^{\circ}$ colonnes du tableau 2$)$, une interprétation en est donnée à la fin de la présente analyse. Pour $T_{\text {MIx i à partir du }}$ $4^{\mathrm{e}}$ jour avant la mesure, on note une diminution de $\mathrm{r}^{2}$ puis une augmentation à partir du $10^{\circ}$ jour. Cette réaugmentation de $\mathrm{r}^{2}$ que l'on retrouve dans le cas des colonnes $T_{\text {MAX i }}$ et $T_{\text {MOY i }}$ du tableau 2 est très probablement d'origine purement climatologique; elle exprime le fait que, statistiquement, le régime thermique naturel de la période considérée a une composante cyclique de périodicité approximativement hebdomadaire (voir aussi GueHL, 1978).

Dans le cas des températures maximales (colonne $T_{\text {MAX }}$, tableau 2), pour les trois jours précédant la mesure, on note une liaison moins importante que dans le cas des températures minimales, par contre les quatre premiers coefficients de

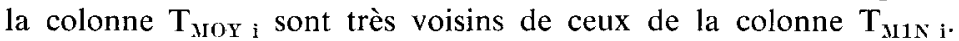




\section{TABleau 2}

Coefficients de corrélation $\left(r^{2}\right)$ des régressions linéaires

liant la capacité photosynthétique du douglas $\left(F_{x}\right.$ y.x $)$ à quelques caractéristiques thermiques des $i$ journées précédentes $(i=1, \ldots, 11)$

Correlation coefficients $\left(r^{2}\right)$ of linear regressions linking the photosynthetic capacity of Douglas-fir $\left(F_{N} u_{x}\right)$ to some thermal characteristics of the $i$ days preceeding the $F_{x}$ uax measurement $(i=1, \ldots, 11)$

\begin{tabular}{|c|c|c|c|c|c|c|}
\hline $\mathrm{i}$ & $\mathrm{T}_{\text {MIN } \mathrm{i}}$ & $\mathrm{T}_{\text {MAX } \mathrm{i}}$ & $T_{\text {Yux }} \mathbf{i}$ & $\mathrm{T}_{\text {MIN 1, i }}$ & et & $\begin{array}{l}T_{\text {MIN } 1, i} \\
T_{\text {MAX } 1, i}\end{array}$ \\
\hline 1 & $0,51 * *$ & $0,28^{*}$ & $0,45 * *$ & $0,51 * *$ & & $0,51^{* *}$ \\
\hline 2 & $0,55^{* *}$ & $0,38 *$ & $0,55 * *$ & $0,64 * *$ & & $0,64 * *$ \\
\hline 3 & $0,62 * *$ & $0,54^{* * * *}$ & $0,63 * *$ & $0,72^{* *}$ & & $0,72 * *$ \\
\hline 4 & $0,35^{*}$ & $0,50^{* *}$ & $0,38 *$ & $0,77^{* *}$ & & $0,77^{* *}$ \\
\hline 5 & 0,22 & $0,38 *$ & $0,37^{*}$ & $0,80^{* *}$ & & $0,80^{* *}$ \\
\hline 6 & 0,06 & 0,24 & 0,21 & $0,83^{* *}$ & & $0,83^{* *}$ \\
\hline 7 & 0,08 & 0,17 & 0,18 & $0,85 * *$ & & $0,85^{* *}$ \\
\hline 8 & 0,10 & 0,16 & 0,15 & $0,87 * *$ & & $0,87 * *$ \\
\hline 9 & 0,06 & $0,34 *$ & 0,22 & $0,87^{* *}$ & & $0,87 * *$ \\
\hline 10 & 0,16 & $0,49 * *$ & $0,38 *$ & $0,87 * *$ & & $0,86^{* *}$ \\
\hline 11 & 0,21 & $0,38^{*}$ & $0,25 *$ & $0,85^{* *}$ & & $0,85^{* *}$ \\
\hline
\end{tabular}

* Significatif au niveau $0,05(14 \mathrm{ddl})$.

** Significatif au niveau 0,01 .

$T_{\text {MIN i }}, T_{M A X}$ et $T_{M O Y Y_{i}}$ sont respectivement les températures journalières minimale, maximale et moyenne du $\mathrm{i}^{\prime}$ jour avant la mesure de $\mathrm{F}_{\mathrm{N} \text { MAx. }} \mathrm{T}_{\text {MIN 1, } \mathrm{i}}$ est une moyenne pondérée des $\mathbf{T}_{\text {MIX } \mathbf{j}}(\mathrm{j}=1, \ldots, \mathrm{i})$ dont l'expression exacte est donnée au 3.2.2.2 [équation (3)]. $\mathrm{T}_{\text {MAX 1, } \mathrm{i}}$ est la même fonction des $\mathrm{T}_{\mathrm{MAX}} \mathrm{j}$.

$T_{M I X}, T_{M A X}, T_{\mathrm{M} O \mathrm{Y}_{i}}$ are respectively the daily minima, maxima and mean temperatures measured $i$ days before $F_{N H A X^{*}} T_{M I N I, i}$ is a ponderated mean value of the $T_{M I N} j(j=1, \ldots, i)$. $A$ detailled expression of $T_{M I N} 1, i$ is given in 3.2.2.2, equation (3). $T_{\text {MAX } 1, i}$ is the same function of the $T_{M A X Y}$

Dans une seconde phase de l'analyse statistique, nous avons considéré non plus des variables climatiques caractérisant individuellement les différentes journées précédant les mesures, mais des variables caractérisant l'ensemble des i journées antérieures aux mesures de $F_{\mathrm{x} \text { Max. }}$. Pour ce type de variables synthétiques ont été considérées dans un premier temps des moyennes pondérées des $T_{\text {MIN } \mathrm{i}}$. Les meilleurs résultats ont été obtenus en donnant à chaque température minimale journalière un poids variant en sens inverse du nombre de jours dont elle précède la mesure de $F_{\text {N MAX }}$. De façon précise, en considérant $\mathrm{i}$ jours avant la mesure, cette pondération avait pour expression : 


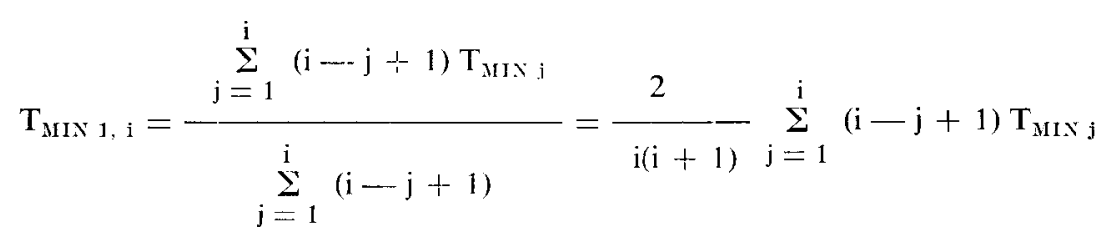

Lorsque l'on fait augmenter $i$ à partir de 1 , le coefficient de corrélation $\mathrm{r}^{2}$ (colonne $T_{\text {MIN 1.; }}$ du tableau 2) augmente dabord rapidement, puis plus lentement, jusqu'à $\mathrm{i}=8\left(\mathrm{r}^{2}=0,87\right)$, puis pour $\mathrm{i}=11, \mathrm{r}^{2}$ amorce une diminution $\left(\mathrm{r}^{2}=0,85\right)$.

L'introduction supplémentaire (régression linéaire double) des températures maximales journalières suivant une pondération identique aux $T_{\text {MIN } 1, i}$ n'améliore pas la liaison (dernière colonne du tableau 2).

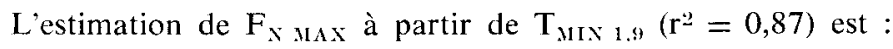

$$
\hat{F}_{\mathrm{XAX}}=1,36 \mathrm{~T}_{\text {MIN } 1,9}+6,29
$$

Les valeurs prises par $\hat{\mathrm{F}}_{\mathrm{N} \text { MAX }}$ lors des différentes journées de mesure sont représentées sur la figure 2 (courbe en trait discontinu dans le bas de la figure). On note une très bonne coincidence dans le sens de variation dans le temps des valeurs observées $F_{X \text { MaX }}$ et des valeurs calculées $\hat{F}_{X \text { Max }}$, cela confirme la bonne valeur

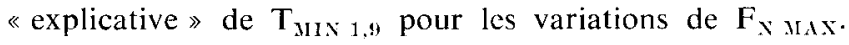

Bien que les mesures relatives aux 15 et 16 mai n'aient pas été considérées dans cette analyse, les valeurs de $\hat{\mathrm{F}}_{\mathrm{X} \text { Max }}$ pour ces deux dates, calculées d'après (3) ont également été représentées sur la figure 2. Ces valeurs sont très nettement supérieures aux valeurs observées $F_{\mathrm{Y} \text { ג }}$, cela met en évidence la particularité de cette période proche du débourrement par rapport à la période hivernale qui la précède.

En conclusion, pour cette période hivernale :

- les effets rémanents du régime thermique sur la capacité photosynthétique s'exercent de façon cumulative décroissante avec une rémanence d'action de l'ordre de 9 jours ;

- les températures minimales semblent jouer un rôle central dans le déterminisme des variations de la capacité photosynthétique.

La première de ces deux caractéristiques fournit une explication au comportement du coefficient de corrélation $\mathrm{r}^{2}$ signalé ci-dessus pour les trois séries de variables $T_{\text {MIN } i}, T_{M A X}$ et $T_{M O Y ~ i}$. Le fait que $r^{2}$ soit maximal pour $i=3$ dans ces trois cas signifie très probablement qu'on optimise alors la relation entre les $T_{M I N ~}, T_{M A X}$ et $T_{M O Y}$ d'une part et $T_{M I N ~ 1.9}$ d'autre part. Ici encore l'explication du comportement de $\mathrm{r}^{2}$ serait donc d'ordre purement climatologique.

\subsection{Seuil thermique de dépression de la capacité photosynthétique}

Si l'analyse statistique permet une bonne aporoche synthétique des relations existant entre la capacité photosynthétique et l'histoire thermique des rameaux, elle ne 
permet pas d'étudier ces relations sous l'angle du déterminisme climatique précis des fluctuations de la capacité photosynthétique. En particulier, existe-t-il, pour le Douglas, un seuil thermique correspondant à un endommagement systématique de la fonction photosynthétique tel que cela a été montré pour d'autres espèces conifères (PISEK, 1973 ; NeILson et al., 1972)?

Dans le cas de notre étude, l'éloignement entre les jours de mesure, le caractère cumulatif des effets rémanents du régime thermique sur $F_{X} M_{X X}$ ainsi que les fluctuations rapides de la température en conditions naturelles empêchent la mise en évidence d'un tel seuil.

Cependant, trois situations particulières méritent d'être examinées, elles concernent les périodes suivantes : 12-19 avril, 27 mars-2 avril, 16-20 mars. Pour chacune de ces trois périodes, on enregistre une chute de la capacité photosynthétique pour des températures de l'air minimales absolues respectivement égales à : $-1{ }^{\circ} \mathrm{C}$, $-1,2{ }^{\circ} \mathrm{C}$ et $-1,8^{\circ} \mathrm{C}$.

Ces observations suggèrent, pour le Douglas, l'existence d'un seuil thermique de dépression de la capacité photosynthétique supérieur ou égal à $-1{ }^{\circ} \mathrm{C}$ sans qu'il soit possible de préciser davantage.

\subsection{Action de la température de mesure sur la photosynthèse nette des rameaux de Douglas}

La réponse de la photosynthèse nette du Douglas à la température «actuelle» $(\theta)$ se caractérise par l'existence d'un large domaine optimal plutôt que par un optimum thermique prononcé. Pour l'ensemble des courbes des figures $1 \mathrm{a}, 1 \mathrm{~b}$ et des courbes obtenues les 2 et 7 avril (figure $1 \mathrm{c}$ ) malgré l'existence d'un optimum thermique peu marqué entre $16^{\circ} \mathrm{C}$ et $20^{\circ} \mathrm{C}$, la photosynthèse reste pratiquement insensible à la température de mesure.

Parallèlement à l'importante dépression de l'activité photosynthétique observée durant la seconde moitié du mois de février (figure 1 a), on note un léger décalage de l'optimum vers les températures élevées. Il est fort probable que ce décalage ne corresponde pas à un déplacement effectif de l'optimum thermique, mais soit lié à une augmentation à court terme de la capacité photosynthétique au cours des mesures, favorisée par l'augmentation de la température de mesure. Une telle évolution a été observée par ailleurs (Schwarz, 1971 ; GUehl, 1978) dans des situations analogues de passage du matériel végétal des basses températures extérieures aux températures plus élevées régnant en laboratoire.

Un changement d'importance dans la forme des courbes $F_{\mathrm{S}}(\theta)$ ne se manifeste qu'à partir du 12 avril (figure $1 \mathrm{c}$ et d) sous la forme d'un affaissement des courbes aux températures supérieures à $15^{\prime \prime} \mathrm{C}$. Cette transformation s'accentue progressivement lorsque l'on se rapproche de la date de débourrement (16 mai) ainsi que le montre l'évolution dans le temps du rapport $R_{z 2}$ de la photosynthèse mesurée à 22 " $C$ à la capacité photosynthétique $F_{N}$ is mesurée le même jour (figure 3 ). De façon concomitante l'optimum thermique de la photosynthèse est décalé vers les basses températures (figure 3 ) et devient inférieur à $10^{\circ} \mathrm{C}$ dès la fin du mois d'avril. 
Les courbes de la figure 4 ont été obtenues toutes deux au 16 mai, date marquant la fin du développement des bourgeons et l'apparition des nouveaux rameaux. La courbe en trait plein a été établie avec des rameaux entiers, la courbe en trait discontinu a été obtenue avec les mêmes rameaux après section et enlèvement des bourgeons. Il apparaît que cette courbe présente une allure analogue à celles obtenues avant le 7 avril avec les rameaux entiers.

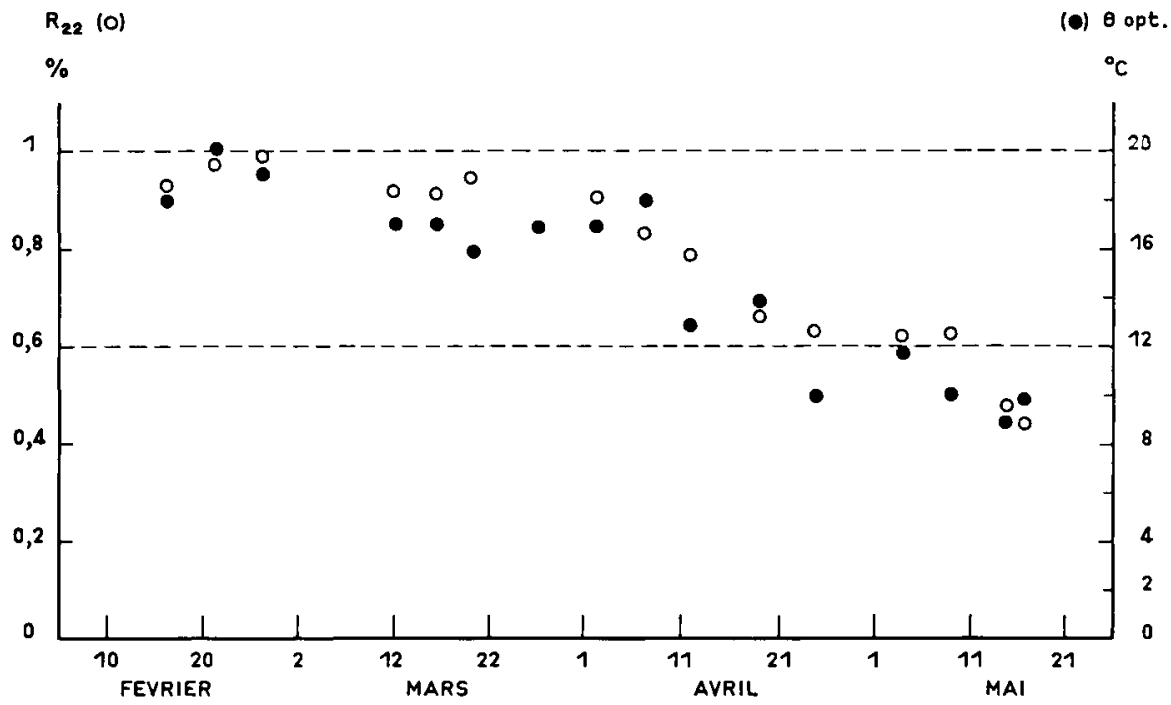

FIG. 3

Du 16-2-79 au 16-5-79, évolution de la température optimale de la photosynthèse nette $\left(\theta\right.$ opt, -) et du rapport $R_{* 2}(0)$ exprimant l'intensité photosynthétique mesurée à $22{ }^{\circ} \mathrm{C}$ relativement à l'intensité $F_{{ }^{*} \text { MAx }}$ correspondant à l'optimum thermique. Le débourrement correspond au 16-5-79

From 2-16th-79 to 5-16th-79 changes of temperature optimum of net photosynthesis $(\theta$ opt, $\bullet)$ and of the $R_{z z}$ ratio $(O)$ giving the photosynthesic rate measured at $22{ }^{\circ} \mathrm{C}$ as percentage of the $F_{x}$ s.s value corresponding to temperature optimum.

Bud sprouting occured on 5-16-79

Sous réserve de l'absence de perturbation de la photosynthèse par la section des bourgeons, la partie comprise entre les deux courbes de la figure 4 correspond à l'activité respiratoire des bourgeons. Cette activité respiratoire, augmentant fortement à partir de $10^{\circ} \mathrm{C}$ serait dans ces conditions responsable de la dépression de la photosynthèse aux températures élevées. Cette dépression commence à se faire sentir entre les 7 et 12 avril 1979 (figure 3). Il est intéressant de noter que le rapport $\mathbf{M S}_{13} / \mathbf{M S}_{\mathrm{R}}$ (tableau 1) présente une augmentation à partir de la même période. Ce parallélisme et les résultats de la figure 4 suggèrent que l'évolution continue dans le temps de la forme des courbes $\mathbf{F}_{\mathrm{N}}(\theta)$ (figure 3 ) soit liée à une augmentation progressive de l'activité respiratoire des bourgeons en développement. 


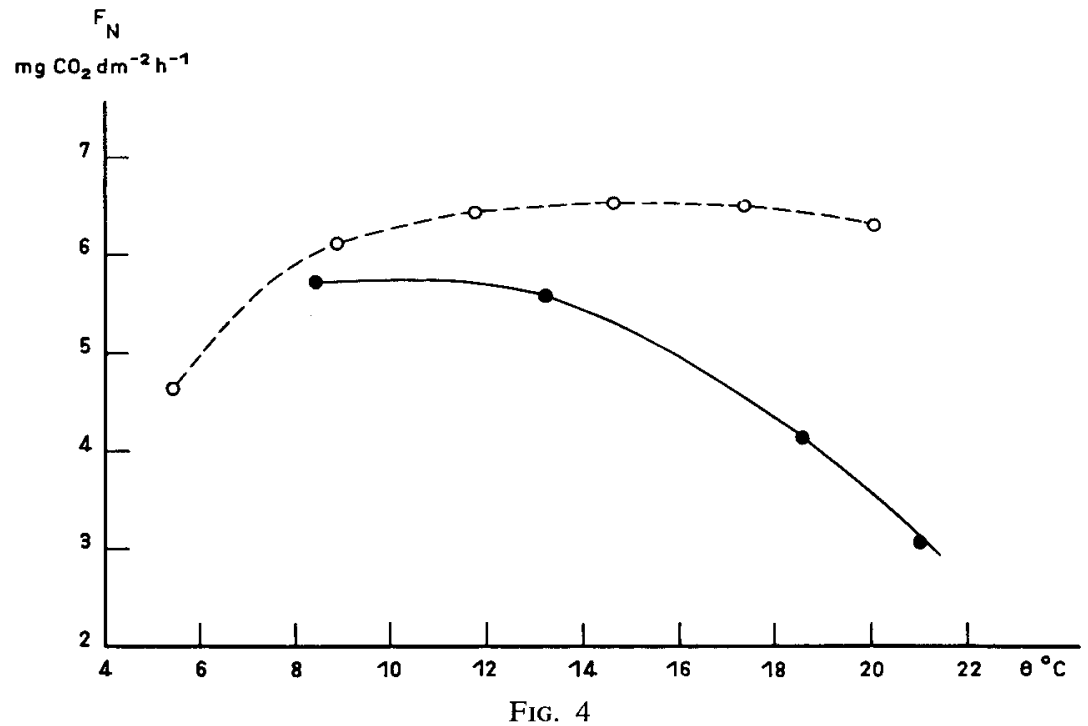

Courbes de réponse de la photosynthèse nette $\left(F_{x}\right)$ à la température de l'air $(\theta)$ obtenues le 16-5-79. Cette date correspond au stade $b_{3}$ du développement des bourgeons (aiguilles de la nouvelle pousse partiellement libérées)

$(\bullet-\bullet)$ : rameaux entiers ; $\left(\bigcirc_{-} \bigcirc\right)$ : mêmes rameaux après ablation des bourgeons.

Response of net photosynthesis $\left(F_{N}\right)$ to air temperature $(\theta)$ etablished on 5-16th-79. That date corresponds to the $b_{s}$ phenologic state in bud development (new needles partially free)

$(\bullet-\bullet)$ : intact shoots; $\left(\mathrm{O}_{-\mathrm{O}}\right)$ : same shoots after bud cutting.

\section{4. - Discussion}

\subsection{Réponse de la photosynthèse à la température}

En dehors de la période proche du débourrement, la photosynthèse du Douglas se caractérise par une très faible sensibilité à la température entre $8{ }^{\circ} \mathrm{C}$ et $24^{\circ} \mathrm{C}$ (figure 1). Un tel comportement a été mis en évidence par plusieurs auteurs pour le Douglas (Salo, 1974 ; Künsle \& Mitscherlich, 1978 ; Guehl, 1978 ; Ducrey, 1981). Malgré cette faible action de la température actuelle on observe un maximum de la photosynthèse entre 16 et $20^{\circ} \mathrm{C}$ (figure 3). Avant le 7 avril cet optimum thermique ne présente pas de décalage significatif.

Divers travaux signalent un ajustement de l'optimum thermique de la photosynthèse nette aux conditions thermiques auxquelles est soumis le végétal. Ce type de résultat est particulièrement net pour certaines études d'acclimatation menées en conditions constantes de phytotron (MoONEy \& HARrison, 1969 ; СнABot \& Lewis, 1976; Strain et al., 1976; Slatyer, 1977). En conditions climatiques naturelles, STrain et al. (1976) notent une diminution très nette (de $25^{\circ} \mathrm{C}$ à $10^{\circ} \mathrm{C}$ ) de l'optimum thermique de Pinus taeda lors du passage de l'été à l'hiver caractérisé par des 
gels fréquents. En zone de montagne du Sud-Est de l'Australie, Slatyer \& Morrow (1977) mettent en évidence un ajustement strict de l'optimum thermique d'Eucalyptus pauciflora à la température maximale moyenne des 10 jours précédant la mesure.

Cependant NeIlson et al. (1972) (Picea sitchensis) ainsi qu'Adams \& FayYaz (1979) (Chrysanthemum morifolium) n'ont pu mettre en évidence de décalage de l'optimum thermique par préconditionnement à différents régimes thermiques de phytotron.

Ces derniers auteurs opposent deux types de stratégies d'adaptation de la photosynthèse aux fluctuations thermiques dans le temps. La première stratégie consiste en un ajustement continu de l'optimum aux conditions thermiques. La seconde stratégie est celle du "généraliste», caractérisée par un large domaine thermique optimal de la photosynthèse et une non plasticité des courbes $F_{N}(\theta)$ face aux fluctuations thermiques.

D’après nos résultats il semblerait que le Douglas puisse être rangé dans la catégorie des "généralistes». NeILSON et al. (1972) signalent un comportement analogue chez Picea sitchensis, autre espèce originaire de la frange côtière pacifique de l'Amérique du Nord.

Aux basses températures, il apparaît (figure 1) que le Douglas présente une légère diminution de la photosynthèse à partir de $10^{\circ} \mathrm{C}$ environ. Pour la même espèce, Salo (1974) décrit un comportement identique, en outre il a mis en évidence un arrêt de la photosynthèse à $-2{ }^{\circ} \mathrm{C}$, la diminution devenant sensible sous $0{ }^{\circ} \mathrm{C}$ seulement.

\subsection{Développement des bourgeons et échanges gazeux de $\mathrm{CO}_{2}$ au niveau du rameau}

Pour la période printanière précédant le débourrement, plusieurs travaux effectués dans des conditions méthodologiques identiques aux nôtres (mesure en conditions contrôlées de laboratoire sur des rameaux coupés de conifères ayant une histoire climatique naturelle) mettent en évidence des transformations dans les échanges gazeux de $\mathrm{CO}_{2}$ au niveau du rameau. Ainsi NeILSON et al. (1972) rapportent une modification dans le temps de la forme des courbes $F_{\mathrm{N}}(\theta)$ très comparable à celle que nous avons décrite au 3.3 (déplacement de loptimum thermique vers les basses températures et dépression de la photosynthèse aux températures élevées). PiseK \& Winkler (1958), SchWarz (1971) ainsi que Fry \& PhILlips (1977) font état d'une diminution de l'activité photosynthétique mesurée au voisinage de $20^{\circ} \mathrm{C}$ à l'approche du débourrement.

Dans notre cas, deux étapes peuvent être distinguées dans les modifications intervenant dans les échanges gazeux de $\mathrm{CO} .$.

- du 12 avril au 9 mai, en relation avec l'activité respiratoire des bourgeons (figure 4) et de leur développement (tableau 1, dernière colonne), on note un changement dans la forme des courbes $F_{N}(\theta)$ (figures 1 et 3 ) sans que la capacité photosynthétique $F_{\mathrm{N} \max }$ soit significativement affectée. En effet, jusqu'au 9 mai, les variations de $\mathrm{F}_{\mathrm{N} \text { Max }}$ suivent le déterminisme climatique explicité au 3.222 (jusqu'à cette date, l'écart $F_{\mathrm{N} \text { MAX }}-\hat{\mathrm{F}}_{\mathrm{N} \text { MaX }}$ est faible); 
- à partir d'une date comprise entre le 9 mai et le 15 mai, soit au maximum 7 jours avant le débourrement ( 16 mai), $F_{\mathrm{N} \text { MAX }}$ devient très nettement inférieur à $\hat{\mathrm{F}}_{\mathrm{N} \text { MAX }}$ (figure 2). Ainsi au $16 \mathrm{mai}$, la capacité photosynthétique des rameaux entiers est de $5,7 \mathrm{mg} \mathrm{CO} \mathrm{dm}^{-2} \mathrm{~h}^{-1}$ (figure 5), celle des mêmes rameaux dépourvus de bourgeons est de $6,6 \mathrm{mg} \mathrm{CO} \mathrm{dm}^{-2} \mathrm{~h}^{-1}$ (figure 4) soit grossièrement la moitié seulement de $\hat{F}_{\mathrm{N} \text { Max }}$ (figure 2). La dépression de $\mathrm{F}_{\mathrm{N} \text { MAX }}$ semble donc ici être essentiellement imputable à des modifications intervenant directement au niveau de l'appareil photosynthétique. Selon FrY \& PhILlips (1977) cette diminution de l'activité photosynthétique serait liée à un changement dans la fonction des chloroplastes qui adopteraient partiellement un fonctionnement d'amyloplastes consistant à accumuler des glucides destinés à l'édification des nouvelles structures. L'étude ultrastructurale menée par SENSER et al. (1975) sur les chloroplastes de Picea abies confirme cette hypothèse.

\subsection{Photosynthèse et histoire thermique des rameaux}

Dans notre étude, la capacité photosynthétique du Douglas oscille en permanence (figure 2) sous l'action d'un jeu d'endommagement-réparation de la fonction photosynthétique dont les mécanismes sont connus (PISEK \& KeMnitzer, 1968 ; LARCher, 1973 ; LeVITt, 1972 ; NeIlson et al., 1972; PiseK, 1973 ; Göring et al., 1978).

Un seuil thermique d'endommagement de la photosynthèse a été mis en évidence pour quelques conifères de la zone tempérée (tableau 3). En hiver ce seuil est inférieur d'environ $3{ }^{\circ} \mathrm{C}$ aux valeurs estivales suite à une réaction adaptative de la plante aux basses températures (endurcissement au froid) (LARCHER, 1973 ; PISEK, 1973 ; Göring et al., 1978 ; ZRALY, 1978).

\section{Tableau 3}

Seuil thermique de dépression de la capacité photosynthétique de quelques conifères de la zone tempérée.

En hiver ce seuil est inférieur de $3^{\circ} \mathrm{C}$ environ aux valeurs estivales

Threshold values of low temperature lasting depressive effects on the photosynthesis of some conifers of the temperate zone.

In winter the values undergo a downward shift of about $3^{\circ} \mathrm{C}$ as compared with summer

\begin{tabular}{|c|c|c|c|}
\hline \multirow{2}{*}{ Auteurs } & \multirow{2}{*}{ Espèce } & \multicolumn{2}{|c|}{ Seuil thermique } \\
\hline & & été & hiver \\
\hline PISEK (1973) & Abies alba & $-3,5^{\circ} \mathrm{C}$ & $-7,5^{\circ} \mathrm{C}$ \\
\hline PISEK (1973) & Picea excelsa & $-4,5^{\circ} \mathrm{C}$ & $-7,5^{\circ} \mathrm{C}$ \\
\hline PISEK (1973) & Pinus silvestris & $-3^{\circ} \mathrm{C}$ & $-7{ }^{\circ} \mathrm{C}$ \\
\hline NEILSON et al. (1972) & Picea sitchensis & $-2{ }^{\circ} \mathrm{C}$ & $-5^{\circ} \mathrm{C}$ \\
\hline
\end{tabular}

En hiver, aucune des espèces du tableau 3 n'est endommagée au-dessus de $-5{ }^{\circ} \mathrm{C}$, alors que pour le Douglas nous avons mis en évidence un endommagement à partir de températures voisines de -1 "C (3.223.). En réalité nous sommes en 
présence de températures de nature différente. Les données du tableau 3 sont des températures d'enceintes fermées dont toutes les parties internes sont en équilibre thermique. Au contraire, nos températures ont été mesurées à l'extérieur, sous abri, or, de nuit, par suite du rayonnement thermique, les températures de surface des végétaux sont inférieures à la température de l'air mesurée sous abri. En conditions de ciel dégagé, cet écart peut atteindre $4{ }^{\circ} \mathrm{C}$ (Aussenac, 1975). Dans l'hypothèse d'un tel écart, le seuil thermique du Douglas se rapproche des valeurs caractérisant les conifères du tableau 3 .

Cette distinction entre deux types de températures constitue une illustration des risques d'une extrapolation abusive de phénomènes observés en laboratoire à la description de la réalité écologique : à Nancy des températures inférieures à $-5^{\circ} \mathrm{C}$ se produisent de façon nettement moins fréquente que des températures inférieures à $-1{ }^{\circ} \mathrm{C}$ (figure 2).

La capacité de «rétablissement » de la photosynthèse après un endommagement dû au froid est un second aspect d'une stratégie d'adaptation aux basses températures (LARCHER, 1973). A cet égard il existe un certain nombre de travaux mettant en évidence des différences entre diverses espèces de conifères. Ainsi le Douglas apparaît comme supérieur à Pinus ponderosa (PHARis et al., 1970), Pinus contorta et Pinus cembra (SCHWARZ, 1971) et dans une moindre mesure à Picea glauca (SCHWARZ, 1971). Dans son aire d'origine (EMmingham \& WARING, 1977) et sous un climat de plaine de la zone tempérée voisin de celui de Nancy (figure 2), cette grande capacité de "rétablissement " lui permet de mettre à profit les périodes de températures clémentes séparant les phases de gel. On notera tout particulièrement sur la figure 2 la spectaculaire augmentation de $F_{N \text { MAX }}$ au début du mois de mars, alors que la température extérieure ne dépasse pas $10^{\circ} \mathrm{C}$.

La valeur maximale de $\mathrm{F}_{\mathrm{N} \text { мax }}\left(12,5 \mathrm{mg} \mathrm{CO}_{2} \mathrm{dm}^{-2} \mathrm{~h}^{-1}\right)$ observée le 25 avril est, quant à elle, de l'ordre de grandeur de la capacité photosynthétique enregistrée pour le Douglas durant une période estivale (données non encore publiées).

\subsection{Contribution à l'étude du fonctionnement photosynthétique hivernal du Douglas suivant le gradient de continentalité en France}

Notre modèle expérimental simple ne saurait refléter la réalité complexe du fonctionnement photosynthétique hivernal in situ. Ce fonctionnement est régi par un ensemble de facteurs agissant de façon interactive (Helms, 1965 ; Pharis et al., 1967 ; Schmidt-Vogt \& Gross, 1976; Künstle \& Mrtscherlich, 1978).

Néanmoins, afin d'approcher globalement l'impact du régime thermique naturel sur le fonctionnement photosynthétique hivernal du Douglas, nous avons mis au point (GueHL, 1978) un index d'assimilation nette, à résolution journalière, pour un rameau de lumière de Douglas supposé être placé en conditions optimales de lumière et d'alimentation hydrique. Cet index est basé sur une modélisation mathématique de la forme des courbes $F_{N}(\theta)$, des relations entre histoire thermique des rameaux et $F_{N}$ ainsi que du fonctionnement respiratoire nocturne des rameaux (pour plus de détails concernant cette modélisation, voir GuEHL, 1978).

Appliqué pour une période hivernale s'étendant du $1^{\text {er }}$ janvier 1978 au 10 avril 1978 à trois stations françaises (Rostrenen en Bretagne, Versailles près de 
Paris et Nancy dans le Nord-Est de la France), cet index met en évidence une péjoration du bilan d'assimilation carbonée hivernale avec la continentalité croissante. Exprimés relativement au bilan calculé pour Rostrenen, les résultats sont :

\begin{tabular}{c|c|c|c}
\hline \hline Station & Rostrenen & Versailles & Nancy \\
\hline Bilan d'assimilation carbonée $\ldots . . .$. & $100 \%$ & $89,5 \%$ & $77,5 \%$ \\
\hline
\end{tabular}

\title{
5. - Conclusion
}

Par la combinaison de mesures en laboratoire et d'un conditionnement in situ du matériel végétal, cette courte étude nous a permis d'aborder le problème des potentialités photosynthétiques hivernales des conifères sous un angle analytique par rapport à l'un de ses facteurs limitants principaux : la température. Nos résultats montrent une variation du potentiel photosynthétique en «dents de scie » caractéristique d'une zone critique de balancement de linfluence de ce facteur limitant entre une situation de repos hivernal total et une situation d'activité photosynthétique faiblement perturbée. Face à une telle sensibilité au régime thermique, on peut penser qu'en France des variations régionales du climat puissent induire des différences de production photosynthétique hivernale chez les conifères.

La modélisation mathématique apparaît comme un outil intéressant pour l'étude de ce type de problème. Il nous semble donc intéressant de progresser dans cette voie en compliquant notre modèle par l'introduction de facteurs supplémentaires (lumière, facteurs hydriques) et par l'étude de leurs interactions sur un modèle expérimental in situ.

D’autre part, parmi les diverses espèces de conifères dont dispose l'aménagiste forestier en France, existe-t-il des différences dans les caractéristiques de l'acclimatation de la fonction photosynthétique aux basses températures (seuil thermique d'endommagement, capacité de « rétablissement» après un coup de froid) ? La mise en évidence de telles différences pourrait contribuer à la mise au point d'indices climatiques de productivité intégrant des particularités écophysiologiques d'espèce ou même de provenance.

Reçu pour publication le 20 octobre 1981.

\author{
Summary \\ Winter photosynthesis of the Douglas-fir (Pseudotsuga menziesii Mirb.) \\ as related to thermal regime
}

In the eastern France, near Nancy, at different days from 02.16th.1979 to 05.16th.1979, shoots of a 19 year old Douglas-fir stand were cut and their net-photosynthesis response to temperature determined in laboratory under light-saturation conditions. 
1. Until beginning of April :

- The response of net-photosynthesis to temperature was characterized by a wide optimal zone centered on $16-18$ " $\mathrm{C}$ rather than by a marked optimum. No significative transformation in the shape of that response curve was observed during the measurement period.

- The photosynthetic capacity is submitted to important changes corresponding to a damage and reparation action on the photosynthetic function linked to the alternation of frost and thaw periods. The after-effects of the thermal regime act upon photosynthesis in a cumulative way and with a 10 day retentivity.

2. From beginning of April to bud-flushing (05.16th)

- In a first time, a more and more important impairment of the $\mathrm{CO}_{2}$ assimilation rate of the shoot was observed at the higher temperatures. Simultaneously the thermal optimum underwent a slight down to $8-10^{\circ} \mathrm{C}$, without the above mentionned climatic determinism of the photosynthetic capacity being significantly affected. That transformation appeared to be due to an increase of the respiratory activity of the developing buds.

- In a second time, a few clays before bud flushing, the photosynthetic capacity decreased in its turn under the influence of physiological changes which seemed to affect the photosynthetic apparatus itself.

The Douglas-fir is characterized by a great capacity of reparation after a cold stress which allows it to turn to account mild temperature winter periods in a climate like that of Nancy. In the temperate zone such an evergreen conifer winter photosynthesis pattern is intermediate between the total winter photosynthetic rest which characterizes the continental and mountain cold winter situations and a pattern without any winter depression as has been observed in mild winter oceanic conditions.

\section{Références bibliographiques}

Adams M.S., Fayyaz M.M., 1979. Temperature acclimation of Net Photosynthesis in relation to growth of a cold hardy chrysanthemum. Ecologia (Berl.), 39, 239-247.

Aussenac G., 1980. Premiers résultats d'une étude de l'influence de l'alimentation en eau sur la croissance des arbres dans un peuplement de douglas (Pseudotsuga menziesii (Mirb.) Franco). R.F.F., XXXII (2), 167-172.

Aussenac G., 1975. Couverts forestiers et facteurs du climat : leurs interactions, conséquences écophysiologiques chez quelques résineux. Station de Sylviculture et de Production, C.N.R.F., Nancy. Thèse d'Etat, 234 p. + annexes.

Bamberg S., Schwarz W., Tranquillini W., 1966. Influence of daylenght on the photosynthetic capacity of stone pine (Pinus Cembra L.). Ecology, 4B (2), 264-269.

Chabot B.F., Lewis A.R., 1976. Thermal acclimation of photosynthesis in northern Red-Oak. Photosynthetica, 10 (2), 130-135.

Chartier P., Bethenod D., 1977. La productivité à l'échelle de la leuille. In : «Les processus de la production végétale primaire », MOYSE A., Gauthier-Villars.

DuCrey M., 1981. Action des basses températures hivernales sur la photosynthèse du cèdre et du douglas. Ann. Sci. for., 38 (3), 317-329.

Emmingham W.H., Waring R.H., 1977. An index of photosynthesis for comparing forest sites in Western Oregon. Can. J. for. Res., 7 (1), 165-174.

Fry D.J., Phillips I.D.J., 1977. Photosynthesis of conifers in relation to annual growth cycles and dry matter production. II. Seasonal photosynthetic capacity and mesophyll ultrastructure in Abies grandis, Picea sitchensis, Tsuga heterophylla and Larix leptolepis growing in S.W. England. Physiol. Plant., 40, 300-306.

Göring H., Thien B.H., Dreier W., Heinke F., Pester A., 1978. Zur Rolle des Prolins in der Reaktion der Pflanzen auf extreme Umweltbedingungen. Math. Nat. R., XXVII (5), 557-560. 
GuEHL J.-M., 1978. Aspect hivernal du fonctionnement photosynthétique du douglas (Pseudotsuga menziesii Mirb.). Station de Sylviculture et de Production, C.N.R.F., Nancy, Document 78/04, 44 p. + annexes.

Hagihara A., Hozumi K., 1977. Studies on photosynthetic production and its seasonal change in a Chamaecyparis obtusa plantation. J. Jpn. For. Soc., 59, 327-337.

Helms J.A., 1965. Diurnal and seasonal patterns of net assimilation in Douglas-fir. Pseudotsuga menziesii (Mirb.) Franco, as influenced by environment. Ecology, 46, 658-708.

KüNstle E., Mitscherlich G., 1978. Photosynthese, Transpiration und Respiration in einem jungen Mischbestand. Angew Bot., 52, 233-252.

Larcher W., 1963. Die Eignung abgeschnittener Zweige und Blätter zur Bestimmung des Assimilationsvermögen. Planta, 56, 575-606.

LARCHER W., 1973. Limiting temperatures for life functions. Gradual progress of damage due to temperature stress. In : Temperature and life (PREcht et al.), Springer Verlag Berlin-Heidelberg-New York, 195-231.

LevitT J., 1972. Responses of plants to environmental stresses. Academic Press, New York, $697 \mathrm{p}$.

MoOney H.A., Harrison A.T., 1969. The influence of conditionning temperature on subsequent temperature-related photosynthetic capacity in higher plants. $1 n$ : Prediction and measurement of photosynthetic productivity. Proceedings of the IGP/PP technical Meetings, Trebon, 14-21 Sept.

Neilson R.E., Ludlow M.M., Jarvis P.G., 1972. Photosynthesis in Sitka spruce (Picea sitchensis (Bong) Carr.). II. Response to temperature. J. appl. Ecol., 9, 721-745.

Pharis R.P., Hellmers H., Schuurmans E., 1967. Kinetics of the daily rate of photosynthesis at low temperatures for two conifers. Plant. Physiol., 42, 525-531.

Pharis R.P., Hellmers H., Schulrmans E., 1970. Effects of subfreezing temperatures on photosynthesis of evergreen conifers under controlled environment conditions. Photosynthetica, 4 (4), 273-279.

PiseK A., Winkler E., 1958. Assimilationsvermögen und Respiration der Fichte (Picea exelsa Link) in verschiedener höhenlage und der Zirbe (Pinus cembra L.) an der alpinen Waldgrenze. Planta, 51, 518-543.

Pisek A., Kemnitzer R., 1968. Der Einfluss von Frost auf die Photosynthese der Weisstanne (Abies alba Mill.). Flora, Abt B., 157, 314-326.

PISEK A., 1973. Effect of temperature on metabolic processes. A. Photosynthesis. In : Temperature and life (Precht H. et al.). Springer-Verlag, Berlin-Heidelberg-New York, 102-127.

Salo D.J., 1974. Factors affecting photosynthesis in Douglas-fir. Ph. D. Thesis, University of Washington, Seattle WA., 131 pages + annexes.

Schmidt-Vogt H., Gross K., 1976. Untersuchungen zum winterlichen Gaswechsel der Fichte (Picea abies (L.) Karst.) unter Freilandbedingungen. Allg. Forst. u. J. Ztg., 147 (10-11), 189-192.

Schwarz W., 1971. Das photosynthesevermögen einiger Immergrüner während des Winters und seine Reaktivierungsgeswindigkeit nach scharfen Frösten. Ber. Dtsch. Bot. Ges. $B d ., 84$, H. 10, 585-594.

SENSER M., Schötz F., Beck E., 1975. Seasonal changes in Structure and Function of Spruce Chloroplasts. Planta, 126, 1-10.

SLATYER R.O., 1977. Altitudinal variation in the photosynthetic characteristics of snow gum. Eucalyptus pauciflora Sieb ex Spreng. III. Temperature response of material grown in contrasting thermal environments. Aust. J. Plant Physiol., 4, 301-312.

SLAtyer R.O., Morrow P.A., 1977. Altitudinal variation in the photosynthetic characteristics of Snow Gum, Eucalyptus pauciflora Sieb. ex Spreng. I. Seasonal changes under Field conditions in the snowy mountains area of South-eastern Australia. Aust. J. Bot., 25, 1-20. 
Strain B.R., Higginbotham K.O., Juliana C. Mulroy, 1976. Temperature preconditionning and photosynthetic capacity of Pinus taeda L. Photosynthetica, 10 (1), 47-53.

Tranquillini W., 1979. Dry matter production of trees at Timberline. In : Physiological ecology of the alpine Timberline, Springer-Verlag, Berlin-Heidelberg-New York, 39-80.

ZelaWSKI W., GoRaL I., 1966. Seasonal changes in the photosynthesis rate of Scots pine (Pinus silvestris L.) seedlings grown from seed of various provenances. Acta Societ. bot. pol., 35, 587-598.

ZELAWSKI W., KUCHARSKA J., 1967. Winter depression of photosynthetic activity in seedlings of Scots Pine (Pinus silvestris L.). Photosynthetica, 1, 207-213.

ZraLY B., 1978. Seasonal changes in frost hardiness and phospholipid content in raspberry canes. Fruit Sci. Rep., V (4), 3-14. 